\title{
Herbivory-induced changes in the olfactory and visual display of flowers and extrafloral nectaries affect pollinator behavior
}

\author{
Mathias Hoffmeister ${ }^{1,2} \cdot$ Robert R. Junker $^{1}$
}

Received: 9 March 2016/Accepted: 14 November 2016/Published online: 25 November 2016

(C) The Author(s) 2017. This article is published with open access at Springerlink.com

\begin{abstract}
Plants communicate with animals by means of multimodal displays and reward mutualistic partners with resources such as nectar. Floral nectar is a key resource for pollinators, whereas extrafloral nectaries (EFNs) support indirect plant defense. Animalpollinated flowers advertise their rewards using modalities such as scent, color and morphology. In EFNs the role of olfactory and visual traits is less well understood. Herbivory has been shown to induce changes in the multimodal display and in resource related characteristics of flowers and EFNs. This may consequently affect the behavior of nectar consumers such as pollinators that occasionally feed on extrafloral nectar in addition to floral nectar. We tested the effect of herbivory (simulated by jasmonic acid treatment) on olfactory, visual and resource related floral and EFN traits in Vicia faba, tracked alterations in bumblebee (Bombus terrestris) preference and evaluated potential outcomes for plant reproduction. In control plants, flowers and EFNs differed in olfactory and visual cues and also in nectar quantity and quality and pollinators clearly preferred to forage on flowers. After jasmonic acid treatment (JA), linalool emissions of EFNs increased in a large proportion of plants, the visual display of EFNs became more salient and nectar volumes increased. In flowers of JA-treated plants we found lower emissions of cinnamic aldehyde and lower sugar concentration in nectar, however visual cues were unaffected. Potentially because of these phenotypic changes bumblebees no longer preferred flowers over EFNs in JA-treated plants. Consequently, this may negatively affect reproductive success in $V$. faba, which is highly dependent on floral visitation for fruit set. Our study adds another aspect to the complexity of plant-pollinator interactions and reveals how herbivory may interfere with plant-pollinator communication. Our findings emphasize that the foraging behavior of
\end{abstract}

Electronic supplementary material The online version of this article (doi:10.1007/s10682-016-9875-y) contains supplementary material, which is available to authorized users.

Robert R. Junker

robert.junker@sbg.ac.at

1 Department of Ecology and Evolution, University Salzburg, 5020 Salzburg, Austria

2 Department of Biology, Institute of Sensory Ecology, University Düsseldorf, 40225 Düsseldorf, Germany 
pollinators not only depends on floral traits, but is affected by the whole plants' multimodal phenotype.

Keywords Indirect defense $\cdot$ Multimodal communication $\cdot$ Mutualism $\cdot$ Plant-pollinator interaction · Resources

\section{Introduction}

Plant-animal interactions range from mutualisms such as pollination to antagonisms such as herbivory. Nectar is a common reward for mutualistic interaction partners, which is either provided by flowers (floral nectar) or at vegetative plant parts (extrafloral nectar). The former usually mediates interactions with pollinators, the latter with natural enemies of herbivores (Heil 2011). Plants advertise floral rewards with multimodal stimuli such as olfactory, visual, tactile and gustatory signals to guide mutualists to the appropriate structure and the same traits are also used to deter non-pollinating flower visitors (Leonard et al. 2012; Junker and Parachnowitsch 2015). Multimodal communication by EFNs has received less attention although visual and olfactory cues have been reported to be associated with EFNs. Few studies suggest volatile emissions from EFNs (Röse et al. 2006; Géneau et al. 2013; Choate and Lundgren 2013), but a detailed analysis of the volatiles emitted by EFNs and their effect on EFN visitors is lacking so far. Similarly, the function of visual traits in interactions with EFNs is largely unknown. EFNs are variable in size and shape, ranging from single pores to structures several centimeters in diameter, and are either colored or indistinguishable from the background (Koptur 1992; Blüthgen and Reifenrath 2003). While access to floral nectar often is restricted by floral morphology, extrafloral nectar is mostly presented as nectar droplets unprotected by plant morphology (Koptur 1992). Moreover, floral nectar production is temporally restricted to the flowering period, whereas extrafloral nectar is produced throughout the growing season on nearly all plant organs (Marazzi et al. 2013). Although the salience, i.e. the perceptual quality of multimodal traits and their conspicuousness against the background (Katzenberger et al. 2013; Schäfer and Ruxton 2015) seems to be higher in flowers than in EFNs, pollinators have regularly been observed visiting EFNs in several legume species (Free 1962; Stoddard and Bond 1987; Waters et al. 2014). Pollinators may be viewed as nectar thieves in these cases, as they exploit resources without contributing to plant defense. Consequently, reduced extrafloral nectar quantities may reduce the efficiency of indirect plant defenses, as less nectar is available for natural enemies (Heil et al. 2004).

Herbivory has been shown to increase the number of EFNs and also the quantity and quality of extrafloral nectar leading to increased recruitment of natural enemies like ants or ladybirds and other visitors which use the nectar as supplementary diet (Pemberton and Vandenberg 1993; Ness 2003; Kost and Heil 2008). So far it has not been investigated whether herbivory also affects olfactory and visual traits of EFNs, although changes in the salience of EFNs may influence interactions with nectar-consumers both qualitatively and quantitatively. Comparable effects have been found in flower-visitor interactions; herbivory-induced changes e.g. in floral scent, morphology and nectar, have been shown to alter flower visitor behavior (Schiestl et al. 2014; Bruinsma et al. 2014; Hoffmeister et al. 2015). Depending on the animal and plant species this resulted in either increased or decreased visitation frequencies (Lucas-Barbosa et al. 2013; Hoffmeister et al. 2015). The 
effects of herbivory on interactions between EFNs and nectar-consumers and on flowervisitor interactions have mostly been studied separately (but see Hernández-Cumplido et al. 2010). However, as pollinators may forage on flowers and EFNs (Free 1962) it is conceivable that herbivory-induced changes in multimodal traits of both nectar sources may affect the pollinators' behavior. An increased visitation frequency by pollinators to EFNs may have consequences for indirect defenses and pollination.

Using Vicia faba L. (Fabaceae) and the pollinator Bombus terrestris L. as study organisms we experimentally tested the following hypotheses. (1) Herbivory (simulated by the application of jasmonic acid) affects olfactory and visual stimuli as well as nectar quantity and quality in flowers and EFNs and (2) herbivory-induced changes of flowers and EFNs affect the behavior of Bombus terrestris, which may alter plant reproductive success. First, we compared the olfactory and visual display of flowers and EFNs and also the volume and the sugar concentration of the nectar sources. Additionally, we tracked the changes in these displays in response to simulated herbivory, i.e. the application of jasmonic acid on plants. In behavioral experiments we tested whether and how bumblebees respond to herbivory-induced changes in both nectar sources. Finally we tested the dependence of $V$. faba on floral visitation for fruit set, to estimate the significance of herbivory-induced changes in pollinator behavior on plant reproduction. Our study thus assesses multimodal signaling in reproductive and vegetative plant traits with complementary ecological functions; pollination and defense. This holistic view of herbivoryinduced alterations in traits and pollinator behavior in the whole-plant context provides novel insights into the relationship between herbivory, pollination and plant defense and reveals interdependencies in the multimodal advertisement of plant resources.

\section{Methods}

\section{Plant cultivation and simulated herbivory treatment}

Vicia faba L. is a self-compatible annual herb with 1-6 stems and alternately arranged compound leaves, producing 50-80 papilionaceous flowers, which are mostly white with a dark-colored spot on the wing petals (Fig. 1a). Starting from the 5th node, flowers are organized in racemes with 2-12 flowers located in each leaf axil. At the base of each compound leaf is a pair of stipules, carrying up to two extrafloral nectaries (EFNs) on their abaxial side visible as dark-colored spots (Fig. 1a; Mondor and Addicott 2003). The number of EFNs and extrafloral nectar production has been shown to vary in response to herbivory and associations of the roots with fungi and bacteria (Laird and Addicott 2007; Jaber and Vidal 2009; Summers and Mondor 2011). In order to standardize the biotic interactions of the plants we controlled for soil biota, growth conditions and excluded herbivorous insects from growth chambers. The substrate (C400, Stender $\odot$, Schermbeck, Germany) was heated for 7 days at $70{ }^{\circ} \mathrm{C}$ in a compartment drier to eliminate the soil biota. Seedlings ("Hangdown", Bingenheimer Saatgut AG, Bingenheim, Germany) germinated for 7 days on wet filter paper and were then inoculated with Rhizobium leguminosarum bv. viceae by turning the radicles in Nodulator ${ }^{\circledR}$ (Becker Underwood Ltd., Littlehampton, UK, $1 \mathrm{~g}$ contains at least $1 \times 10^{9}$ viable cells). Seedlings were transferred into $2 \mathrm{~L}$ pots filled with the prepared soil and raised under fluorescent tubes (Lumilux Cool Daylight L58W/865, Osram, München, Germany) in a growth chamber with a day/night rhythm (16 h of light at $20{ }^{\circ} \mathrm{C} ; 8 \mathrm{~h}$ of darkness at $\left.12{ }^{\circ} \mathrm{C}\right)$. Plants were watered daily. To 
exclude root herbivores a non-systemic insecticide (Stechmückenfrei, Neudorff, Emmerthal, Germany) was added to the water.

Herbivory was simulated by application of jasmonic acid (JA), a plant hormone involved in wound response that has been shown to affect the physiology of EFNs (Heil et al. 2001) and whose derivatives have been shown to induce defense responses in $V$. faba (Birkett et al. 2000). For treatments, all leaves were sprayed until run-off with a $1 \mathrm{mM}$ aqueous JA-solution containing 0.5\% Tween20 (JA from Biomol GmbH, Hamburg, Germany; Tween 20 from Roth $\mathrm{GmbH}$, Karlsruhe, Germany). Control plants were treated with the same solution, containing no JA.

\section{Olfactory cues of flowers and EFNs}

We sampled volatiles of flowers and EFNs and tracked potential herbivory-induced changes in scent emissions. As plant damage would have affected scent bouquets, we sampled the organs without picking them or damaging surrounding tissues. In $V$. faba stipules (bearing EFNs) and flowers are tightly attached to the stem and the leaves, making it impossible to sample them separately without damaging the plant. For EFN scent we sampled extracted extrafloral nectar only, for flowers we determined floral scent emissions indirectly by using data from whole plant samples, leaf samples, EFN samples and the literature. First, we collected volatiles from the upper part of whole plants including multiple inflorescences, leaves and EFNs (whole plant sample) and then sampled volatiles of picked leaves and of extracted extrafloral nectar. From the whole plant samples part of the compounds were directly assigned to the floral bouquet as they had previously been described as floral scents in V. faba (Griffiths 1999). From the remaining compounds we added those to the floral bouquet that were not found in the samples of picked leafs or EFNs.

For the whole plant samples, we enclosed the upper third of the plants $\left(n_{\mathrm{C}}=6\right.$, $n_{\mathrm{JA}}=13$ ) in oven bags (Toppits, Minden, Germany); empty oven bags (air samples) were used as ambient control. Subsequently, we cut a hole at the bottom of the flower bags, installed traps filled with Poropak-Q (ARS, Inc, Gainesville, USA) at the top of the flower bags and then constantly pulled air from flower bags (flow rate: $1000 \mathrm{~mL} \mathrm{~min}^{-1}$, sampling: $60 \mathrm{~min}$ ) as described in Hoffmeister et al. (2015). Volatiles were eluted with $150 \mu \mathrm{L}$ dichloromethane. By means of an AOC-20i autoinjector (Shimadzu, Tokyo, Japan) $1 \mu \mathrm{L}$ of the eluate was injected at $220^{\circ} \mathrm{C}$ (split ratio 1:1) into a Shimadzu GC-MS-QP2010 Ultra (Shimadzu, Tokyo, Japan) equipped with ZB-5 fused silica column (5\% phenyl polysiloxane; $30 \mathrm{~m}$ long, inner diameter $0.32 \mathrm{~mm}$, film thickness $0.25 \mu \mathrm{m}$, Phenomenex). Column flow (carrier gas: helium) was set to $3 \mathrm{~mL} \mathrm{~min}^{-1}$. The $\mathrm{GC}$ oven temperature started at $40{ }^{\circ} \mathrm{C}$, was then increased to $220^{\circ} \mathrm{C}\left(10{ }^{\circ} \mathrm{C} \mathrm{min}^{-1}\right)$ and held for $2 \mathrm{~min}$. The MS interface was kept at $250{ }^{\circ} \mathrm{C}$ and the ion source at $200{ }^{\circ} \mathrm{C}$. Mass spectra were taken at $70 \mathrm{eV}$ (in EI mode) from $\mathrm{m} / \mathrm{z} 30$ to 530.

For leaf scent, picked leaves from control and JA-treated plants $\left(n_{\text {plants }}=12\right)$ were transferred into oven bags, which were tightly closed for scent accumulation (30 min); again empty oven bags were used as control. After scent accumulation, volatiles were pulled from the oven bags and trapped on $1.5 \mathrm{mg}$ of Tenax-TA (mesh 60-80; Supelco, Bellafonte, USA) and $1.5 \mathrm{mg}$ Carbotrap B (mesh 20-40) in a quartz vial (Varian Inc., Palo Alto, USA; length $15 \mathrm{~mm}$, inner diameter $2 \mathrm{~mm}$ ) for $30 \mathrm{~s}$ (flow rate: $200 \mathrm{~mL} \mathrm{~min}^{-1}$ ). Traps were placed in an automatic thermal desorption system (model TD-20, Shimadzu, Tokyo, Japan, split ratio 1:1) coupled with a similar GC-MS, with the same column model and model specifications as described above, except that column flow was set to 
$1.5 \mathrm{~mL} \mathrm{~min}^{-1}$, GC oven temperature was increased to $250{ }^{\circ} \mathrm{C}\left(6^{\circ} \mathrm{C} \mathrm{min}{ }^{-1}\right)$ and held for $1 \mathrm{~min}$ and the MS interface was kept at $220{ }^{\circ} \mathrm{C}$.

For EFN scent, all available extrafloral nectar per plant from control and JA-treated plants $\left(n_{\mathrm{C}}=12, n_{\mathrm{JA}}=16\right)$ was collected by absorbing the nectar droplets with filter paper (diameter $15 \mathrm{~mm}$, Rotilabo, Roth, Karlsruhe, Germany). The filter papers were immediately transferred into oven bags for scent accumulation (180 $\mathrm{min})$ and subsequently volatiles were trapped and analyzed as described for leaves; nectar-free dry filter papers were used as control. Filter papers and also leaves were handled with forceps that were cleaned with hexane and acetone before and after handling to remove any apolar or polar residues.

We processed GC/MS data with the GCMSolution package, Version 2.72. Compounds were identified, comparing them to mass spectra and retention indices of commercially available standard substances or to compounds listed in mass spectral libraries Wiley Registry 9th Edition, NIST 2011 and FFNSC2. For analyses, we calculated the proportion of each compound contributing to the total scent emission by dividing the integrated peak area of the compound by the total peak area per sample. For multivariate patterns in the proportional scent composition of flowers and EFNs of control and JA-treated plants we calculated Euclidean distances between scent samples, and visualized these distances in a non-metric multidimensional scaling (NMDS) plot using the vegan package in R. To compare substances shared by flowers and EFNs and assess the effect of simulated herbivory on the proportional emission of single compounds we performed Wilcoxon rank-sum tests.

\section{Visual cues (dark-colored spots) of flowers and EFN}

Plants evolved visual cues like colored nectar guides to attract pollinators and specifically guide them to floral rewards (Sprengel 1793; Leonard and Papaj 2011) and a similar function has been hypothesized for colored EFNs (Koptur 1992). Pollinators have a preference for dark guides (Biesmeijer et al. 2005), and show a bias towards large floral cues (Naug and Arathi 2007). In order to examine visual cues of flowers and EFNs and to track herbivory-induced changes, we measured the area [ellipse: $\pi \times(0.5$ length $\times 0.5$ width $)$ ] of the dark-colored spots on petals and stipules occurring next to flowers or flower buds on the oldest stem of control and JA-treated plants using a caliper rule. Measurements were performed on 5-6 week old plants that had been subjected to control and JA-treatment weekly as described above, starting 2-3 weeks after germination (2-4 leaf stage, $\left.n_{\mathrm{C}}=15, n_{\mathrm{JA}}=18\right)$. For statistical analyses we compared the spot areas of the flowers and EFNs between the treatments using two-sided Wilcoxon rank-sum tests (unpaired design) and within plants between flowers and EFNs using two-sided Wilcoxon signed-rank tests (paired design).

\section{Number of nectaries, nectar volume and nectar sugar concentration}

To compare floral and extrafloral nectar rewards and assess the effect of herbivory on nectar traits we used a different set of flowering plants (6-7 weeks old). We first removed all nectar standing crop and subsequently treated plants comparable in number of flowers and EFNs (flowers: $W=122, p=0.27$; EFNs: $W=81.5, p=0.20$, two-sided Wilcoxon rank-sum tests) once with control or JA-solution as described above ( $n=15$ per treatment). Three days later we quantified nectar volumes and nectar sugar concentrations of all flowers and EFNs occurring next to flowers or flower buds on the oldest stem. Nectar droplets were collected using microcapillaries $(5 \mu \mathrm{L}$, A. Hartenstein, Würzburg Germany) and sugar concentration was measured with a handheld refractometer (A. Krüss Optronic, Hamburg, Germany). A refractometer measures the refractive index of liquids, which is proportional to the sugar 
concentration in aqueous solutions. For statistical analyses we calculated means per plant (e.g. mean floral nectar volume) and compared the nectar parameters of flowers and EFNs between the treatments using two-sided Wilcoxon rank-sum tests (unpaired design) and within plants between flowers and EFNs using two-sided Wilcoxon signed-rank tests (paired design).

\section{Pollinator preferences for flowers or EFNs as a function of herbivory}

To examine the foraging choices of pollinators towards flowers and EFNs as a function of herbivory, we tested the behavior of laboratory-reared Bombus terrestris L. (re-natur GmbH, Ruhwinkel, Germany). Bumblebee colonies were kept in cardboard nest boxes connected to a transparent Perspex flight cage (cage dimensions $0.4 \times 0.4 \times 0.8 \mathrm{~m}$, width $\mathrm{x}$ length $\mathrm{x}$ height), illuminated with fluorescent tubes (Lumilux Cool Daylight L58W/865, Osram, München, Germany, $30 \mathrm{kHz}$ flicker). Bumblebees were fed with a $30 \%$ sucrose solution by transparent, unscented $5 \mathrm{~mL}$ plastic syringes in the flight cage accessible to flying individuals only. Honeybee-collected pollen was offered in the dark directly in the nest boxes of the colonies. In behavioral experiments, we observed single initially naïve bumblebees (i.e. they never visited flowers or EFNs before) foraging on flowers and EFNs of control and JA-treated plants offered in pairs in a flight cage $\left(n_{\text {bumblebees }}=74, n_{\text {colonies }}=4, n_{\text {plant pairs }}=15\right.$, plants were treated as described above 1 week before testing). The flight cage (width $\times$ length $\times$ height: $0.6 \times 0.4 \times 0.8 \mathrm{~m}$ ) was covered with gauze to allow for gas exchange (mesh size $1 \times 1 \mathrm{~mm}$ ) and illuminated as described above. Bumblebees rarely managed to open flowers of $V$. faba on their first floral visit, however we counted the attempt to enter a flower as an intentional choice. In EFNs we counted only those visits as intentional choices where we observed the bumblebees probing or drinking the extrafloral nectar (proboscis extension). Each bumblebee was tested separately and only once. Each plant was tested with 3-5 bumblebee individuals in total. For each bumblebee individual we recorded all choices within a single foraging bout; from the first visit of a flower/EFN of a control or JA-treated plant until leaving it. We scored the bumblebee's behavior as "leaving the plant" if the bumblebees did not continue to forage on the first plant but switched to the second plant or randomly flew in the flight cage without returning to the first plant. We found no preference for control or JA-treated plants, when examining bumblebee's first choice (Wilcoxon signedrank test: $n_{\text {plant pairs }}=15, V=43.5, p=0.36$ ), thus for the statistical analyses we focused on the within plant choices between flowers and EFNs. We used two-sided Wilcoxon signedrank tests to test for bumblebee's first choice per plant (i.e. we tested the proportion of bumblebees choosing flowers first versus the proportion of bumblebees choosing EFNs first per plant) and to compare the overall preference per plant (proportion of choices for flowers or EFNs within a single foraging bout). Analyses were performed for control and JA-treated plants separately.

\section{Plant reproduction}

In order to test $V$. faba's dependency on flower visitation for reproduction we performed pollination experiments on plants $(n=10)$ grown in the Botanical Garden of the University of Düsseldorf. Each plant was subjected to three treatments on separate stems; open visitation, hand-pollination and visitor exclusion. First, developing flower buds ( $n=11-18$ for each stem) were marked with a string and enclosed with a net (mesh size $1 \times 1 \mathrm{~mm}$ ) until flowering. For the open visitation treatment we removed the net during anthesis of flowers. For the hand-pollination treatment we opened the net, provided all flowers with pollen from the same plant and reclosed the net to exclude further visitors. For the exclusion treatment the net was 
opened and immediately reclosed to control for handling. After anthesis we counted all developing fruits from marked flowers. For statistical analyses we performed a FriedmanANOVA with post hoc paired Wilcoxon tests ( $\alpha=0.05$ adjusted by false discovery rate) using proportional fruit set (fruits per number of flowers) as response and treatment as explanatory variable adding plant as error to account for the nested design.

All analyses were performed with R 3.1.2 (R Core Team 2014).

\section{Results}

\section{Olfactory cues of flowers and EFNs}

Flowers and EFNs largely differed in the number and identity of compounds emitted and their proportional composition (Table 1). The floral scent bouquet (19 compounds) was dominated by linalool (main compound), trans- $\beta$-ocimene, $\alpha$-pinene and limonene; other mono- and sesquiterpenes and some aromatics were found in lower proportions (Table 1). The scent bouquet of EFNs (6 compounds) comprised benzaldehyde, benzyl alcohol, linalool, octanol and two unidentified compounds. A large proportion of compounds emitted from EFNs was also found in leaves (Table 1). Floral and EFN scent were clearly separated in a NMDS plot, however JA-treatment had no effect on multivariate patterns (Supplementary material, figure S1). Linalool was the only compound emitted by both flowers and EFNs, but the floral bouquet contained a higher proportion of this compound than the extrafloral nectar bouquet, irrespective of the treatment (Fig. 1b; Table 1, Wilcoxon rank-sum tests, $n=6-16$ plants, $W \geq 72, p<0.001)$. A higher percentage of JAtreated plant individuals emitted linalool from EFNs compared to control plants (C: $33.3 \%$, JA: $62.5 \%$ ), albeit on average the proportional emission did not differ significantly between control and treatment plants (Fig. $1 \mathrm{~b}$; Table $1, n_{\mathrm{C}}=12, n_{\mathrm{JA}}=16, W=55$, $p=0.052$ ). Flowers decreased the proportional cinnamic aldehyde emission after JAtreatment (Table $1, n_{\mathrm{C}}=6$ plants, $n_{J A}=12$ plants, $W=65, p=0.02$ ); other compounds were not affected ( $n=6-16$ plants, $W \geq 18, p \geq 0.07$ ).

\section{Visual cues (dark-colored spots) of flowers and EFN}

The area of dark-colored spots was significantly larger in flowers compared to EFNs in control and in JA-treated plants, respectively (Fig. 1c, two-sided Wilcoxon signed-rank test; $n_{\mathrm{C}}=12$ plants, $V=78, p<0.001 ; n_{\mathrm{JA}}=14$ plants, $\left.V=91, p<0.001\right)$. Simulated herbivory did not affect spot areas in flowers, but increased the spot areas in EFNs twofold (Fig. 1c, two-sided Wilcoxon rank-sum test; flowers: $n_{\mathrm{C}}=12$ plants, $n_{\mathrm{JA}}=13$ plants, $W=88, p=0.61$, EFNs: $n_{\mathrm{C}}=15$ plants, $n_{\mathrm{JA}}=15$ plants, $\left.W=13, p<0.001\right)$.

\section{Number of nectaries, nectar volume and nectar sugar concentration}

Flowers were generally less numerous than EFNs in control as well as in JA-treated plants (Fig. 1d, two-sided Wilcoxon signed-rank test; $n_{\mathrm{C}}=14$ plants, $V=105, p<0.01$; $n_{\mathrm{JA}}=14$ plants, $\left.V=105, p<0.01\right)$; treatment had no effect on the number of nectaries (Fig. 1d, two-sided Wilcoxon rank-sum test; flowers: $n_{\mathrm{C}}=14$ plants, $n_{\mathrm{JA}}=14$ plants, $W=122, p=0.27$, EFNs: $n_{\mathrm{C}}=15$ plants, $n_{\mathrm{JA}}=15$ plants, $\left.W=81.5, p=0.20\right)$. The proportion of flowers and EFNs that produced nectar ('active' nectaries) did not differ in 


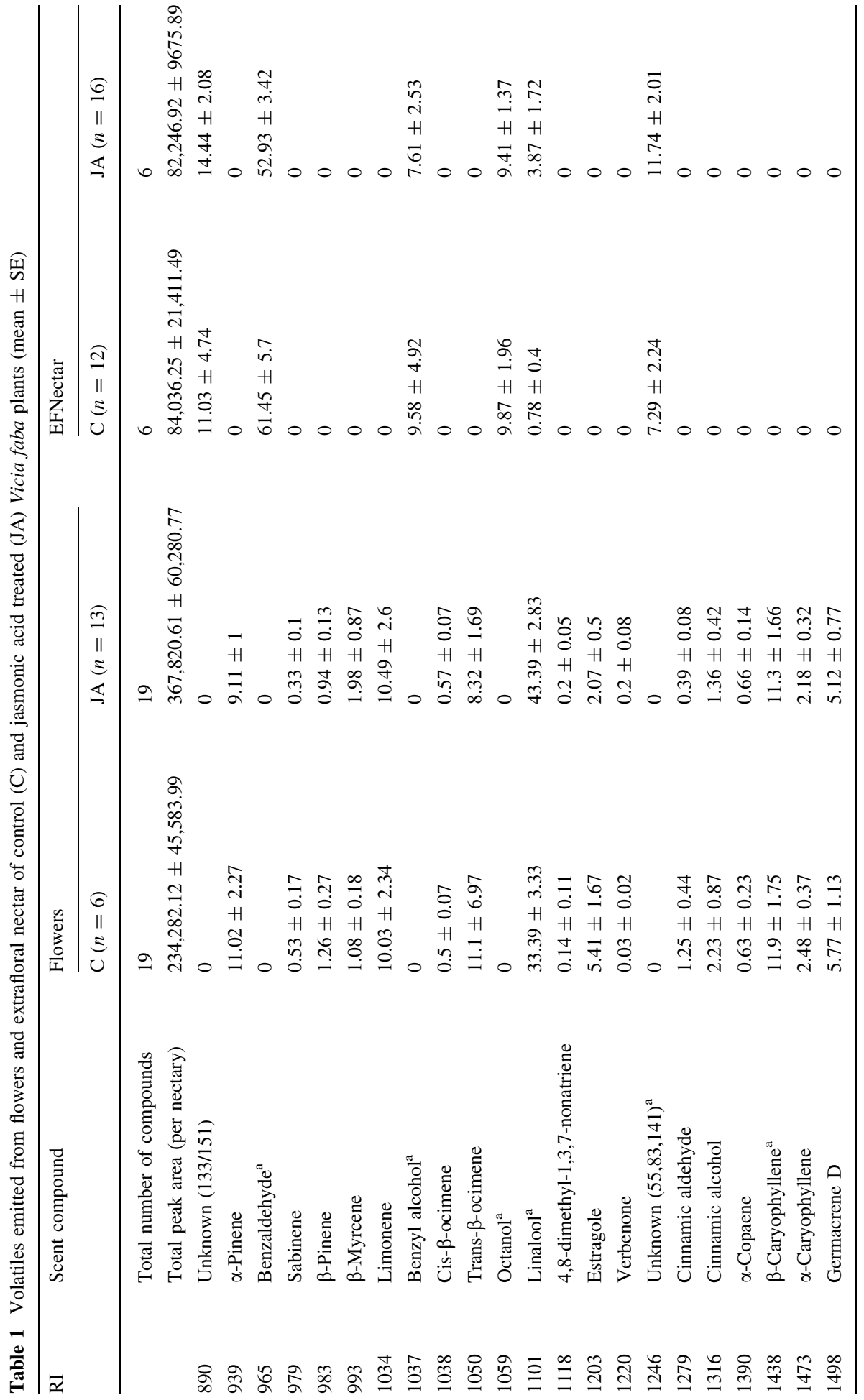




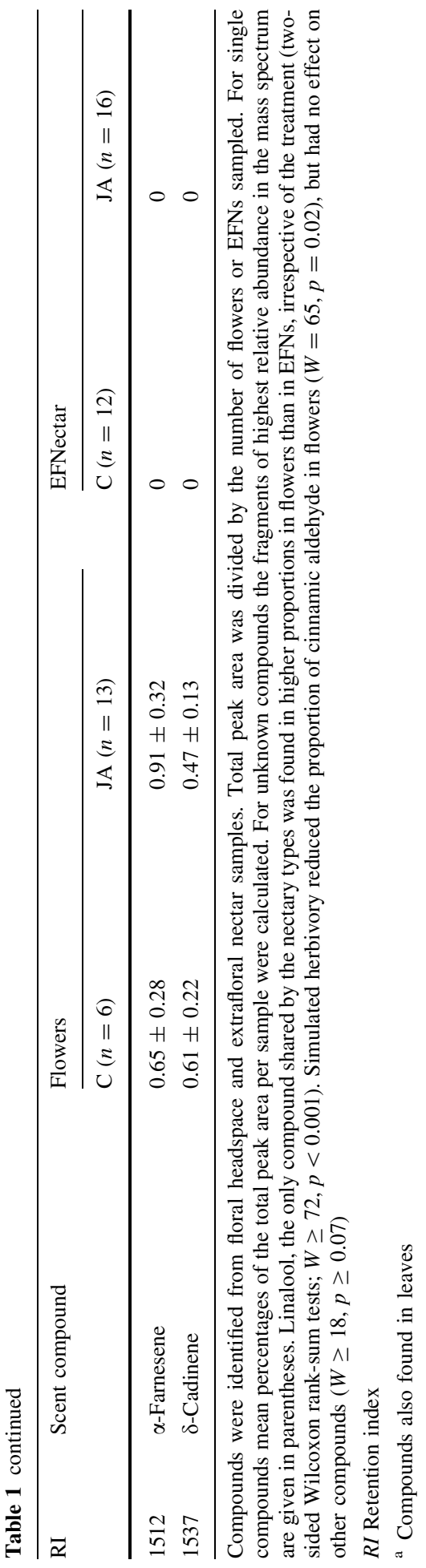




\section{(a)}

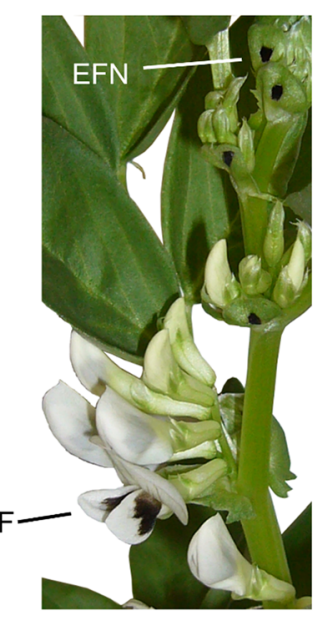

(f)

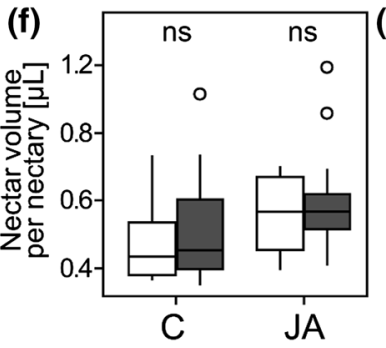

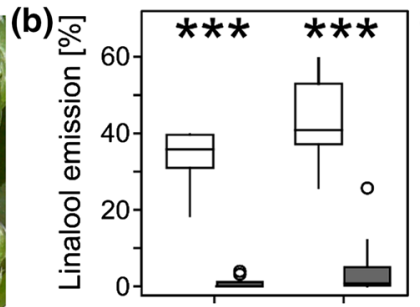

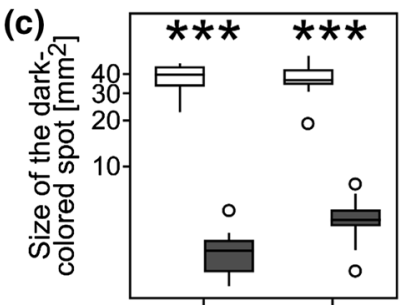

(d)
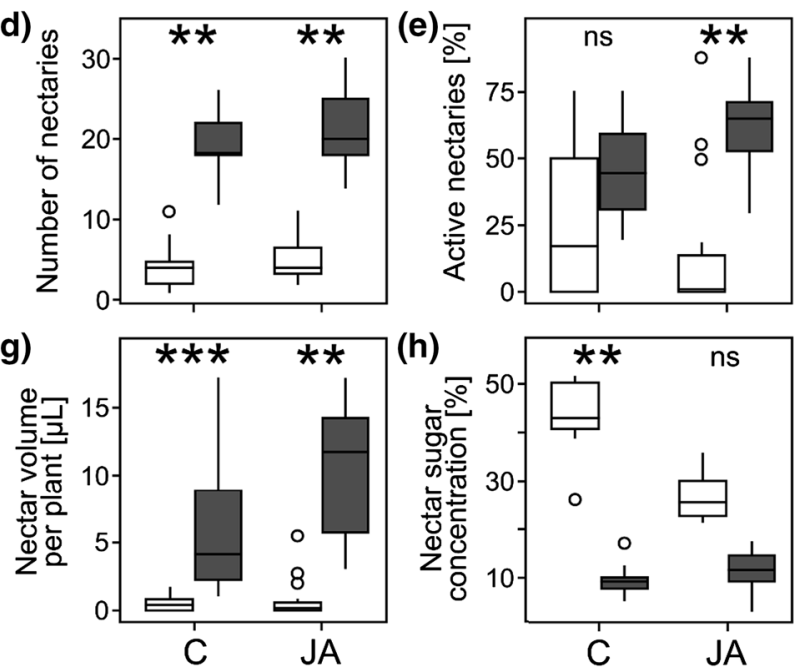

(h)

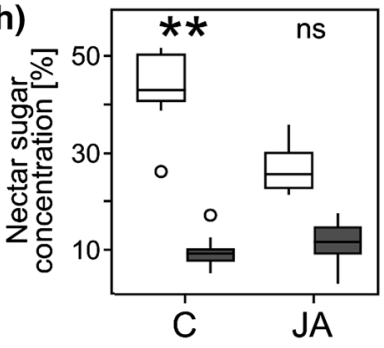

Fig. 1 Olfactory and visual properties and nectar traits of flowers (white boxplots) and extrafloral nectaries (EFNs, grey boxplots) in control (C) and jasmonic-acid treated (JA) plants of Vicia faba. a Location of flowers and EFNs. Boxplots (median, quartile, range) are shown for $\mathbf{b}$ the proportional linalool content in floral headspace and extrafloral nectar samples, $\mathbf{c}$ the area of the dark-colored spot on petals and stipules, d the total number of flowers and EFNs in the upper plant part, e the percentage of flowers and EFNs that actively secreted nectar ('active' nectaries) and $\mathbf{f}$ the mean nectar volume per single nectary, $\mathbf{g}$ the total nectar volume of flowers and EFNs per plant, $\mathbf{h}$ the mean nectar sugar concentration per single nectary. Asterisks denote significant differences between nectary types after two-sided Wilcoxon signed-rank tests. Detailed statistics and statistics for comparisons between treatments are given in the text. $* * * p<0.001$; $* * p<0.01, n s$ not significant

control plants, but we found a higher proportion of 'active' EFNs versus 'active' flowers in JA-treated plants (Fig. $1 \mathrm{e} ; n_{\mathrm{C}}=14$ plants, $V=70, p=0.09 ; n_{\mathrm{JA}}=14$ plants, $V=87$, $p<0.01)$. Simulated herbivory did not affect the proportion of 'active' flowers, whereas it resulted in a higher proportion of 'active' EFNs in JA-treated versus control plants (Fig. 1e, flowers: $n_{\mathrm{C}}=14$ plants, $n_{\mathrm{JA}}=14$ plants, $W=126, p=0.17$; EFNs: $n_{\mathrm{C}}=15$ plants, $n_{\mathrm{JA}}=15$ plants, $\left.\mathrm{W}=51, p=0.011\right)$. On average, 'active' flowers and EFNs offered similar nectar volumes in control and in JA-treated plants (Fig. 1f, $n_{\mathrm{C}}=9$ plants, $V=23, p=1 ; n_{\mathrm{JA}}=4$ plants, $\left.V=9, p=0.25\right)$, and the nectar volume of single flowers or EFNs did not differ between the treatments (Fig. 1f, flowers: $n_{\mathrm{C}}=9$ plants, $n_{\mathrm{JA}}=4$ plants, $W=12, p=0.41$; EFNs: $n_{\mathrm{C}}=15$ plants; $n_{\mathrm{JA}}=15$ plants, $W=67, p=0.06$ ). In total, we found larger extrafloral nectar volumes compared to floral nectar volumes per plant in control and in JA-treated plants (Fig. $1 \mathrm{~g}, n_{\mathrm{C}}=14$ plants, $V=105, p<0.001$; $n_{\mathrm{JA}}=14$ plants, $\left.V=104, p<0.01\right)$. JA-treatment, however, induced higher secretion rates in EFNs but not in flowers (Fig. $1 \mathrm{~g}$, flowers: $n_{\mathrm{C}}=14$ plants, $n_{\mathrm{JA}}=14$ plants, 
$W=119, p=0.31$; EFNs: $n_{\mathrm{C}}=15$ plants, $n_{\mathrm{JA}}=15$ plants, $\left.W=49, p<0.01\right)$. In control plants the nectar sugar concentration was higher in flowers than in EFNs, in JAtreated plants the nectar sugar concentration did not differ between the nectary types (Fig. $1 \mathrm{~h}, n_{\mathrm{C}}=9$ plants, $V=0, p<0.01 ; n_{\mathrm{JA}}=4$ plants, $V=0, p=0.125$ ). Simulated herbivory reduced the floral sugar nectar concentration but the extrafloral nectar sugar concentration was unaffected (Fig. 1 h, flowers: $n_{\mathrm{C}}=9$ plants, $n_{\mathrm{JA}}=4$ plants, $W=34$, $p=0.017$; EFNs: $n_{\mathrm{C}}=15$ plants, $n_{\mathrm{JA}}=15$ plants, $W=67, p=0.06$ ).

\section{Pollinator preferences for flowers or EFN as a function of herbivory}

Bumblebees regularly visited EFNs in flight cage experiments (33\% of a total of 664 visits on either flowers or EFNs). Most bumblebee individuals foraged either on flowers or on EFNs per trial, i.e. if they started to forage on one of the resources they usually continued to visit the same resource. On the individual plant level, there was no consistent pattern when examining the foraging decisions of bumblebees, sometimes they chose the same resource as the preceding bumblebee, sometimes they made different choices. On average the number of visits per bee did not differ between control and JA-treated plants (Mean \pm SE, control plants: $8.7 \pm 1.2$, JA-treated plants: $11.1 \pm 1.3$; two-sided Wilcoxon rank-sum test, $n=74$ bumblebees, $W=543, p=0.13$ ). Comparing the first choices of bumblebees (i.e. the proportion of bumblebees choosing flowers first versus the proportion of bumblebees choosing EFNs first per plant) we found a preference for flowers over EFNs in control plants (Fig. 2a, two-sided Wilcoxon signed-rank tests; $n=15$ plants, first choice: $V=9, p=0.017)$. This preference remained unchanged when examining the overall preference (all visits within a single foraging bout, Fig. $2 b, V=12.5, p=0.021$ ). In JA-treated plants bumblebees, however, showed no preferences for flowers over EFNs and visited flowers and EFNs in similar proportions (Fig. 2a, b, $n=15$ plants, first choice: $V=21, p=0.53$, overall preference: $V=39, p=0.25$ ).
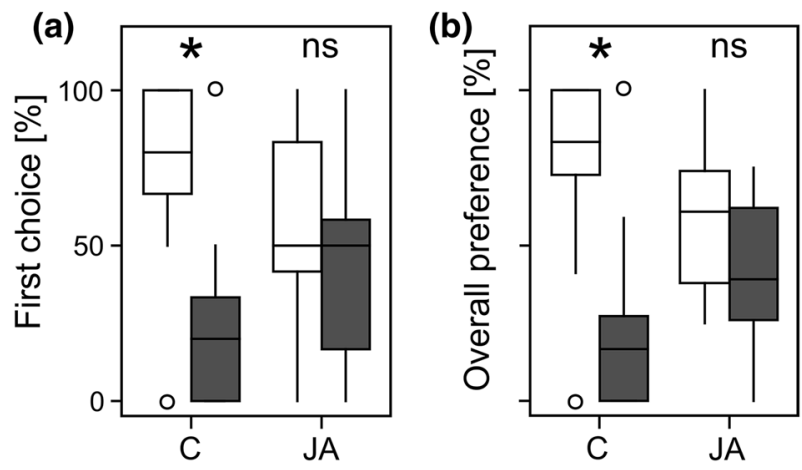

Fig. 2 Preference of Bombus terrestris for flowers (white boxplots) or EFNs (grey boxplots) foraging on control (C) and jasmonic acid-treated plants (JA) of Vicia faba. Boxplots (median, quartile, range) are shown for a the first choice of bumblebees for flowers or EFNs (proportion of bumblebees choosing flowers or EFNs first, per plant), b the overall preference (proportion of visits towards flowers and EFNs within a foraging bout). Asterisks denote significant differences after two-sided Wilcoxon signed-rank tests. ${ }^{*} p<0.05$, ns not significant 


\section{Plant reproduction is dependent on pollinator visitation}

Fruit set in $V$. faba was significantly higher in the hand-pollinated treatment (mean $\pm \mathrm{SE}$, $8.29 \pm 3.19 \%)$ and the open visitation treatment $(11.61 \pm 3.75 \%)$ compared to the visitor exclusion treatment where no fruits developed (Friedman ANOVA: $\chi_{2}^{2}=8.91, p=0.011$ ).

\section{Discussion}

In this study we examined visual and olfactory cues and nectar rewards of flowers and EFNs in $V$. faba. In undamaged plants flowers and EFNs differed in visual and olfactory display and offered nectar of distinct quality and quantity. Pollinators preferred to forage on flowers, potentially because flowers were more attractive than EFNs. After jasmonic acid treatment the visual and olfactory display of EFNs became more salient, i.e. the darkcolored spots on the stipules became larger and EFNs tended to emit more linalool. In contrast, visual stimuli of flowers (the dark-colored spots) did not strongly respond to JAtreatment; olfactory stimuli changed in a trace compound only. Thus, flowers and EFNs converged in their conspicuousness. Presumably due to these changes in the phenotype of EFNs, bumblebees showed equal preference for either flowers or EFNs, both in their first choice and when examining the overall preference (all visits during a single foraging bout). Reduced flower visitation may negatively affect reproductive success in $V$. faba, as fruit set turned out to depend on flower visitation.

Although few studies have indirectly shown that EFNs are scented (Röse et al. 2006; Géneau et al. 2013; Choate and Lundgren 2013), this is to our knowledge the first study that describes the volatile composition of EFNs. The volatiles found in extrafloral nectar were mainly a subset of compounds emitted by leaves, which is comparable to the situation in floral nectar scent which often originates from surrounding floral tissues (Raguso 2004; Kessler and Baldwin 2007). Most extrafloral nectar compounds may have been passively absorbed by the aqueous nectar (Raguso 2004), however, we found an unidentified compound, which was not part of the leaf bouquet. Either it was actively secreted into the extrafloral nectar (Kessler and Baldwin 2007) or its concentration in the leaf bouquet was below the detection threshold. Due to methodological constraints we restricted scent sampling to extracted extrafloral nectar, however the whole EFN structure potentially emits volatiles, which may be investigated in future studies. The extrafloral nectar scent bouquet was less complex and of a different composition compared to the floral scent bouquet, which may reflect the different ecological functions of the organs. Extrafloral nectar scent was dominated by benzaldehyde, a compound that has been shown to attract pollinators (Theis 2006), but also natural enemies of herbivores like coccinellids or parasitic wasps (Han and Chen 2002; Jones et al. 2011), which supports the defense function of EFNs. Floral scent contained mainly mono- and sesquiterpenes known to attract pollinators and deter antagonists simultaneously (Junker and Blüthgen 2010). The major compound linalool is common to flowers, but also involved in herbivory-induced responses (Du et al. 1998; Han and Chen 2002; Knudsen et al. 1993).

In a multivariate analysis we found no clear effect of simulated herbivory on the composition of floral or extrafloral nectar scent of $V$. faba. However, pollinators have been shown to often use single floral compounds for foraging decisions (Junker 2016). We found that JA-treated plants, in contrast to control plants, increased the emission of linalool in EFNs. Naïve bumblebees are attracted to linalool and readily learn to associate it with a 
sugar reward (Junker and Blüthgen 2010; Katzenberger et al. 2013). Other studies demonstrated that changes in the emission rates of linalool have the potential to increase the salience of resources for bees (Sandoz et al. 2001; Wright and Smith 2004). Based on these findings it is conceivable that increased linalool emission rates from EFNs may lead to increased salience of these resources and thus more frequent visits by bumblebees.

Assuming that the visual salience of flowers and EFNs is positively correlated to the size of the spots on petals and stipules, flowers of undamaged $V$. faba plants are more salient to bumblebees than EFNs. JA-treatment did not affect petal spots, but led to a twofold increase in EFN spot areas, thus potentially increasing the detectability of EFNs, which may have additionally contributed to the observed shifts in pollinator preferences from flowers to EFNs in JA-treated plants.

In general EFNs were more numerous than flowers leading to higher extrafloral nectar volumes per plant. Herbivory treatment had no effect on the nectar volumes of single 'active' flowers and single 'active' EFNs (but compare to Heil et al. 2001; Radhika et al. 2010), however we found a higher proportion of 'active' EFNs in JA-treated plants, resulting in higher total extrafloral nectar volumes compared to control plants.

Meanwhile, the floral nectar sugar concentration decreased in JA-treated compared to control plants, suggesting a resource trade-off between defense and reproduction. Potentially, in JA-treated plants the floral nectar sugar concentration was reduced in order to allocate it to 'active' EFNs (but see Chamberlain and Rudgers 2012). At the same, reallocation of nectar sugar may help the plants to move natural enemies to prefer EFNs over flowers and thus avoid floral nectar robbing.

Besides nectar volume and sugar concentration recent reviews suggest that the quality of floral and extrafloral nectar, i.e. the composition of sugars, proteins and secondary metabolites may favor different visitors, thus enabling the plant to attract mutualists and defend against antagonists simultaneously (González-Teuber and Heil 2009; Heil 2011). Herbivory-induced changes in nectar properties may alter these patterns. For instance, increases in extrafloral nectar volumes are known to enhance the number of ants foraging for this sugar source (Ness 2003). Herbivory-induced plant responses may also concern other beneficial insects, e.g. coccinellids or spiders, which regularly feed on EFNs in addition to their prey (Pemberton and Vandenberg 1993; Lundgren 2009).

Our study reveals that herbivory-induced changes in nectar and in multimodal plant stimuli apart from flowers also affect pollinator behavior. The herbivory-induced increased salience of EFNs (larger visual display, trend towards higher scent emission) potentially increased EFN visits by naïve bumblebees. The increased extrafloral nectar volumes in JAtreated compared to control plants may have stimulated further EFN visits. In control plants bumblebees strongly preferred flowers over EFNs, whereas in JA-treated plants we found no preferences, neither in bumblebee's first choice nor when examining the overall preference (all visits within a single foraging bout). Though scent marks may have affected bumblebee behavior (Saleh et al. 2006), it is unlikely that they played a major role in our study. If scent marks played a role we would expect bumblebee individuals to show either consistently similar or contrasting behavior to individuals tested immediately prior, reflecting either attraction or repulsion to scent marks, respectively. Bees did, however, not show such behavior. As we found only small herbivory-induced changes in flowers but strong responses in EFNs we assume that altered pollinator behavior was mainly driven by changes in EFNs. Disentangling the relative contribution of herbivory-induced changes in olfactory and visual traits and associated nectar rewards of both nectaries to the observed pollinator behavior would be an interesting field for future studies. So far most studies have focused on flower traits to assess the effect of herbivory on flower visitor behavior, and 
showed either increased or decreased flower visitation rates depending on the animal and plant species (Lucas-Barbosa et al. 2013; Hoffmeister et al. 2015). Our study investigating herbivory-induced changes in flowers and EFNs thus adds another aspect to plant-mediated interactions of herbivores and pollinators.

We found reduced flower visitation rates in JA-treated compared to control plants which may reduce plant reproductive success in $V$. faba, which depends on flower visits for fruit set (compare to Lord and Heslop-Harrison 1984). Herbivory-altered pollinator behavior towards EFNs and flowers may thus have profound effects on other plant-animal interactions. For instance, pollinators that feed on extrafloral nectar may reduce resources for natural enemies, which has been shown to reduce indirect defense in Macaranga tanarius (Heil et al. 2004). Therefore, pollinators consuming extrafloral nectar may indirectly benefit herbivores. Herbivory-induced multimodal changes in EFNs may thus be associated with two drawbacks; reduced pollination and reduced defense. However, note that we worked on $V$. faba plants cultivated in a growth chamber free from ants or other EFN visitors and we conducted behavioral assays with naïve bumblebees. In the field, these patterns may not be as pronounced, especially when other consumers of extrafloral nectar compete with pollinators such as ants that aggressively defend their resources (Ness 2003).

Our findings contribute to integrative studies on plant-pollinator and plant-herbivore interactions (Lucas-Barbosa 2016) and shed new light on how herbivory may affect pollinator behavior and thus pollination. We found that (simulated) herbivory may not only induce changes in extrafloral nectar production (Heil et al. 2001; Ness 2003), but also affects olfactory cues (linalool emission) and visual cues (area of the dark-colored spot) associated with these rewards. Herbivory-induced changes in these cues presumably evoked responses in pollinator behavior in behavioral assays leading to higher visitation rates to EFNs by pollinators. Our study supports the notion that pollinators primarily forage for resources, not flowers (Junker and Parachnowitsch 2015). They adjusted their foraging behavior using further cues in addition to floral traits, especially when herbivory came into play. Thus, for a comprehensive understanding about the ecology and evolution of plantpollinator mutualisms, interactions at the phenotype of whole plants including vegetative and floral plant parts should be considered.

Acknowledgements Open access funding provided by Paris Lodron University of Salzburg. We thank Sina Kimberly Hirnstein and Pia Carina Höftmann for help with the experiments and Klaus Lunau, Nico Blüthgen, Stefan Dötterl, Irmgard Schäffler, Sarah Papiorek and Petra Wester for logistic support and discussions.

Funding This study was funded by the Deutsche Forschungsgemeinschaft (DFG JU2856/2-2).

\section{Compliance with ethical standards}

Conflict of interest The authors declare that they have no conflict of interest.

Open Access This article is distributed under the terms of the Creative Commons Attribution 4.0 International License (http://creativecommons.org/licenses/by/4.0/), which permits unrestricted use, distribution, and reproduction in any medium, provided you give appropriate credit to the original author(s) and the source, provide a link to the Creative Commons license, and indicate if changes were made. 


\section{References}

Biesmeijer JC, Giurfa M, Koedam D et al (2005) Convergent evolution: floral guides, stingless bee nest entrances, and insectivorous pitchers. Naturwissenschaften 92:444-450

Birkett MA, Campbell CAM, Chamberlain K et al (2000) New roles for cis-jasmone as an insect semiochemical and in plant defense. Proc Natl Acad Sci USA 97:9329-9334

Blüthgen N, Reifenrath K (2003) Extrafloral nectaries in an Australian rainforest: structure and distribution. Aust J Bot 51:515-527

Bruinsma M, Lucas-Barbosa D, ten Broeke CJM et al (2014) Folivory affects composition of nectar, floral odor and modifies pollinator behavior. J Chem Ecol 40:39-49

Chamberlain SA, Rudgers JA (2012) How do plants balance multiple mutualists? Correlations and pollinators in cotton (Gossypium). Evol Ecol 26:65-77

Choate BA, Lundgren JG (2013) Why eat extrafloral nectar? Understanding food selection by Coleomegilla maculata (Coleoptera: Coccinellidae). Biocontrol 58:359-367

Du Y, Poppy GUYM, Powell W et al (1998) Identification of semiochemicals released during aphid feeding that attract parasitoid Aphidius ervi. J Chem Ecol 24:1355-1368

Free JB (1962) The behaviour of honeybees visiting beans (Vicia faba). J Anim Ecol 31:497-502

Géneau CE, Wäckers FL, Luka H et al (2013) Effects of extrafloral and floral nectar of Centaurea cyanus on the parasitoid wasp Microplitis mediator: olfactory attractiveness and parasitization rates. Biol Control 66:16-20

González-Teuber M, Heil M (2009) Nectar chemistry is tailored for both attraction of mutualists and protection from exploiters. Plant Signal Behav 4:809-813

Griffiths D (1999) Epicuticular waxes and volatiles from faba bean (Vicia faba) flowers. Phytochemistry 52:607-612

Han B, Chen Z (2002) Behavioral and electrophysiological responses of natural enemies to synomones from tea shoots and kairomones from tea aphids, Toxoptera aurantii. J Chem Ecol 28:2203-2219

Heil M (2011) Nectar generation, regulation and ecological functions. Trends Plant Sci 16:191-200

Heil M, Koch T, Hilpert A et al (2001) Extrafloral nectar production of the ant-associated plant, Macaranga tanarius, is an induced, indirect, defensive response elicited by jasmonic acid. Proc Natl Acad Sci USA 98:1083-1088

Heil M, Hilpert A, Krüger R et al (2004) Competition among visitors to extrafloral nectaries as a source of ecological costs of an indirect defence. J Trop Ecol 20:201-208

Hernández-Cumplido J, Benrey B, Heil M (2010) Attraction of flower visitors to plants that express indirect defence can minimize ecological costs of ant-pollinator conflicts. J Trop Ecol 26:555-557

Hoffmeister M, Wittköpper N, Junker RR (2015) Herbivore-induced changes in flower scent and morphology affect the structure of flower-visitor networks but not plant reproduction. Oikos 125:1241-1249

Jaber LR, Vidal S (2009) Interactions between an endophytic fungus, aphids and extrafloral nectaries: do endophytes induce extrafloral-mediated defences in Vicia faba? Funct Ecol 23:707-714

Jones VP, Steffan SA, Wiman NG et al (2011) Evaluation of herbivore-induced plant volatiles for monitoring green lacewings in Washington apple orchards. Biol Control 56:98-105

Junker RR (2016) Multifunctional and diverse floral scents mediate biotic interactions embedded in communities. In: Blande JD, Glinwood RT (eds) Deciphering chemical language of plant communication. Signaling and communication in plants. Springer, Heidelberg

Junker RR, Blüthgen N (2010) Floral scents repel facultative flower visitors, but attract obligate ones. Ann Bot 105:777-782

Junker RR, Parachnowitsch AL (2015) Working towards a holistic view on flower traits-How floral scents mediate plant-animal interactions in concert with other floral characters. J Indian Inst Sci 95:44-67

Katzenberger TD, Lunau K, Junker RR (2013) Salience of multimodal flower cues manipulates initial responses and facilitates learning performance of bumblebees. Behav Ecol Sociobiol 67:1587-1599

Kessler D, Baldwin IT (2007) Making sense of nectar scents: the effects of nectar secondary metabolites on floral visitors of Nicotiana attenuata. Plant J 49:840-854

Knudsen JT, Tollsten L, Bergström LG (1993) Floral scents—a checklist of volatile compounds isolated by head-space techniques. Phytochemistry 33:253-280

Koptur S (1992) Extrafloral nectary-mediated interactions between insects and plants. In: Bernays E (ed) Insect-plant interactions, vol IV. CRC Press, London, pp 81-129

Kost C, Heil M (2008) The defensive role of volatile emission and extrafloral nectar secretion for lima bean in nature. J Chem Ecol 34:2-13

Laird RA, Addicott JF (2007) Arbuscular mycorrhizal fungi reduce the construction of extrafloral nectaries in Vicia faba. Oecologia 152:541-551 
Leonard AS, Papaj DR (2011) ' $X$ ' marks the spot: the possible benefits of nectar guides to bees and plants. Funct Ecol 25:1293-1301

Leonard AS, Dornhaus A, Papaj DR (2012) Why are floral signals complex? An outline of functional hypotheses. In: Patiny S (ed) Evolution of plant-pollinator relationships. CUP, Cambridge

Lord EM, Heslop-Harrison Y (1984) Pollen-stigma interaction in the leguminosae: stigma organization and the breeding system in Vicia faba L. Ann Bot 54:827-836

Lucas-Barbosa D (2016) Integrating studies on plant-pollinator and plant-herbivore interactions. Plant Sci 21:125-133

Lucas-Barbosa D, van Loon JJA, Gols R et al (2013) Reproductive escape: annual plant responds to butterfly eggs by accelerating seed production. Funct Ecol 27:245-254

Lundgren JG (2009) Relationships of natural enemies and non-prey foods. Springer, Dordrecht

Marazzi B, Bronstein JL, Koptur S (2013) The diversity, ecology and evolution of extrafloral nectaries: current perspectives and future challenges. Ann Bot 111:1243-1250

Mondor EB, Addicott JF (2003) Conspicuous extra-floral nectaries are inducible in Vicia faba. Ecol Lett 6:495-497

Naug D, Arathi HS (2007) Receiver bias for exaggerated signals in honeybees and its implications for the evolution of floral displays. Biol Lett 3:635-637

Ness JH (2003) Catalpa bignonioides alters extrafloral nectar production after herbivory and attracts ant bodyguards. Oecologia 134:210-218

Pemberton RW, Vandenberg NJ (1993) Extrafloral nectar feeding by ladybird beetles (Coleoptera: Coccinellidae). Proc Entomol Soc Wash 95:139-151

Radhika V, Kost C, Boland W et al (2010) The role of jasmonates in floral nectar secretion. PLoS One $5: \mathrm{e} 9265$

Raguso RA (2004) Why are some floral nectars scented? Ecology 85:1486-1494

R Core Team (2014) R: a language and environment for statistical computing. R Foundation for Statistical Computing, Vienna

Röse USR, Lewis J, Tumlinson JH (2006) Extrafloral nectar from cotton (Gossypium hirsutum) as a food source for parasitic wasps. Funct Ecol 20:67-74

Saleh N, Ohashi K, Thomson JD et al (2006) Facultative use of the repellent scent mark in foraging bumblebees: complex versus simple flowers. Anim Behav 71:847-854

Sandoz JC, Pham-Deleguè MH, Renou M et al (2001) Asymmetrical generalisation between pheromonal and floral odours in appetitive olfactory conditioning of the honey bee (Apis mellifera L.). J Comput Physiol A 187:559-568

Schäfer HM, Ruxton GD (2015) Signal diversity, sexual selection and speciation. Annu Rev Ecol Evol Syst 46:573-592

Schiestl FP, Kirk H, Bigler L et al (2014) Herbivory and floral signaling: phenotypic plasticity and tradeoffs between reproduction and indirect defense. New Phytol 203:257-266

Sprengel CK (1793) Das entdeckte Geheimnis der Natur im Bau und der Befruchtung der Blumen. Vieweg, Berlin

Stoddard F, Bond D (1987) The pollination requirements of the faba bean. Bee World 68:144-152

Summers M, Mondor EB (2011) Rhizobium alters inducible defenses in broad bean, Vicia faba. Open J Ecol $1: 57-62$

Theis N (2006) Fragrance of canada thistle (Cirsium arvense) attracts both floral herbivores and pollinators. J Chem Ecol 32:917-927

Waters T, Chirikian D, Carmona-Galindo VD (2014) Insect visitation of peduncular and petiolar extrafloral nectar glands on castor bean (Ricinus communis L.) plants in Southern California. J Evol Biol Res 6:5-8

Wright GA, Smith BH (2004) Different thresholds for detection and discrimination of odors in the honey bee (Apis mellifera). Chem Senses 29:127-135 